\title{
OPEN Humic + Fulvic acid mitigated Cd adverse effects on plant growth, physiology and biochemical properties of garden cress
}

\author{
Ertan Yildirim $^{1 凶}$, Melek Ekinci $^{1}$, Metin Turan ${ }^{2 \varpi}$, Güleray Ağar ${ }^{3}$, Atilla Dursun ${ }^{1,4}$, Raziye Kul ${ }^{1}$,
} Zeynep Alim ${ }^{1}$ \& Sanem Argin $^{5}$

Cadmium (Cd) is a toxic and very mobile heavy metal that can be adsorbed and uptaken by plants in large quantities without any visible sign. Therefore, stabilization of $\mathrm{Cd}$ before uptake is crucial to the conservation of biodiversity and food safety. Owing to the high number of carboxyl and phenolic hydroxyl groups in their structure, humic substances form strong bonds with heavy metals which makes them perfect stabilizing agents. The aim of this study was to determine the effects of humic and fulvic acid (HA + FA) levels $(0,3500,5250$, and $7000 \mathrm{mg} / \mathrm{L})$ on alleviation of Cadmium (Cd) toxicity in garden cress (Lepidium sativum) contaminated with $\mathrm{Cd}\left(\mathrm{CdSO}_{4} \cdot 8 \mathrm{H}_{2} \mathrm{O}\right)(0,100$, and $200 \mathrm{Cd} \mathrm{mg} / \mathrm{kg})$ under greenhouse conditions. Our results showed that, $\mathrm{Cd}$ stress had a negative effect on the growth of garden cress, decreased leaf fresh, leaf dry, root fresh and root dry weights, leaf relative water content (LRWC), and mineral content except for Cd, and increased the membrane permeability (MP) and enzyme (CAT, SOD and POD) activity. However, the HA + FA applications decreased the adverse effects of the Cd pollution. At $200 \mathrm{mg} / \mathrm{kg}$ Cd pollution, HA + FA application at a concentration of $7000 \mathrm{mg} / \mathrm{L}$ increased the leaf fresh, leaf dry, root fresh, root dry weights, stem diameter, leaf area, chlorophyll reading value (CRV), MP, and LRWC values by $262 \%, 137 \%, 550 \%, 133 \%, 92 \%, 104 \%$, $34 \%, 537 \%$, and $32 \%$ respectively, compared to the control. Although the highest $\mathrm{H}_{2} \mathrm{O}_{2}, \mathrm{MDA}$, proline and sucrose values were obtained at $200 \mathrm{mg} / \mathrm{LCd}$ pollution, $\mathrm{HA}+\mathrm{FA}$ application at a concentration of $7000 \mathrm{mg} / \mathrm{L}$ successfully alleviated the deleterious effects of $\mathrm{Cd}$ stress by decreasing $\mathrm{H}_{2} \mathrm{O}_{2}$, MDA, proline, and sucrose values by $66 \%, 68 \%, 70 \%$, and $56 \%$, respectively at $200 \mathrm{mg} / \mathrm{kg}$ Cd pollution level. $\mathrm{HA}+\mathrm{FA}$ application at a concentration of $7000 \mathrm{mg} / \mathrm{L}$ successfully mitigated the negative impacts of Cd pollution by enhanced $\mathrm{N}, \mathrm{P}, \mathrm{K}, \mathrm{Ca}, \mathrm{Mg}, \mathrm{Fe}, \mathrm{Mn}, \mathrm{Cu}, \mathrm{Mn}, \mathrm{Zn}$, and $\mathrm{B}$ by $75 \%, 23 \%, 84 \%, 87 \%, 40 \%$, $85 \%, 143 \%, 1 \%, 65 \%$, and $115 \%$, respectively. In addition, $\mathrm{HA}+\mathrm{FA}$ application at a concentration of $7000 \mathrm{mg} / \mathrm{L}$ successfully reduced $\mathrm{Cd}$ uptake by $95 \%$ and $\mathrm{Cl}$ uptake by $80 \%$. Considering the plant growth parameters, the best results were determined when HA + FA concentration was $7000 \mathrm{mg} / \mathrm{L}$. We have shown that, it is critical to apply a humic substance with high percentage of $F A$, which was $10 \%$ in this study, to mitigate the adverse effects of heavy metal stress on plant growth. In conclusion, the application of HA + FA may be suggested as an effective solution for reducing the Cd uptake of the plants by stabilizing $\mathrm{Cd}$ in soil and preventing translocation of $\mathrm{Cd}$ from the roots of plant to its shoot and leaves.

Soil and water are valuable natural resources crucial for the sustainability of agriculture. However, anthropogenic activities severely damaged and contaminated both soil and water. One of the major problems is the discharge of sewage water and industrial wastes to agricultural areas which creates various threats by polluting the environment with heavy metals, salts, and nitrates ${ }^{1}$. Pollution caused by heavy metals is a serious problem

\footnotetext{
${ }^{1}$ Department of Horticulture, Faculty of Agriculture, Atatürk University, 25240 Erzurum, Turkey. ${ }^{2}$ Department of Genetics and Bioengineering, Yeditepe University, 34755 Istanbul, Turkey. ${ }^{3}$ Department of Biology, Faculty of Science, Atatürk University, 25240 Erzurum, Turkey. ${ }^{4}$ Department of Horticulture and Agronomy, Kyrgyz-Turkish Manas University, Bishkek, Kyrgyz Republic. ${ }^{5}$ Department of Agricultural Trade and Management, Yeditepe University, 34755 Istanbul, Turkey. ${ }^{\circledR}$ email: ertanyil@atauni.edu.tr; m_turan25@hotmail.com
} 
since contamination of the food chain by metal pollutants pose a danger to plant, animal and human health due to the high toxicity ${ }^{2}$.

Plants have a natural tendency to uptake various metals through their roots. Some of them are basic plant micronutrients such as $\mathrm{Cu}^{2+}, \mathrm{Co}^{2+}, \mathrm{Fe}^{2+}, \mathrm{Mo}^{2+}, \mathrm{Mn}^{2+}$, and $\mathrm{Zn}^{2+}$, while several such as $\mathrm{Hg}^{2+}, \mathrm{Cd}^{2+}, \mathrm{Ni}^{2+}$, and $\mathrm{Pb}^{2+}$ can be detrimental to plants. Impacts of metals on plants vary from genotype to genotype ${ }^{3}$ and the toxicity depends on ion type, ion concentration, plant species, and plant growth stage $e^{4,5}$.

Among the pollutants, commonly referred to as "heavy metal", cadmium (Cd) is a major problem due to its mobility in the plant-soil system. Although $\mathrm{Cd}$ is naturally present in the environment in a small amount (0.04-0.32 mM), agricultural applications of pesticides, phosphate fertilizers, fallout and biosolids from nonagricultural industry have enriched the soil with this element ${ }^{6}$. Cd is very mobile and is easily adsorbed by plants ${ }^{7}$. $\mathrm{Cd}$ is particularly dangerous since plants grown in contaminated soils may adsorb Cd in large quantities without any visible signs ${ }^{3}$ and enter the human body by feeding. ${ }^{8}$. Therefore, low Cd intake by plants and the physiological responses of plants to $\mathrm{Cd}$ pollution are crucial to the conservation of biodiversity and food safety.

Humic substances are important organic materials that directly and indirectly affect plant growth, improve the physical and chemical characteristics of soil and increase vegetative production. The solubility and bioavailability of these substances change, once they form compounds with metals. The strong bonds formed between humic substances and toxic heavy metal ions play an important role in plant production in terms of mitigating the negative effects of heavy metal stress on plant development ${ }^{9}$.

Leafy vegetables are high metal ion accumulators when compared with other vegetable species ${ }^{10}$. Lepidium sativum, (a.k.a. garden cress), is a leafy vegetable that grows mainly in temperate regions. Since ancient times, seeds, leaves, roots, and flowers of garden cress have been used to treat various diseases or ailments ${ }^{11}$. Earlier reports have pointed out that cress is sensitive to cadmium $(\mathrm{Cd})$ stress. However, to our best knowledge there is no study investigating the effect of HA + FA applications on the cress grown under Cd stress. Thus, in this work, $\mathrm{CdSO}_{4} \cdot 8 \mathrm{H}_{2} \mathrm{O}$ polluted soil $(0,100$, and $200 \mathrm{mg} \mathrm{Cd} / \mathrm{kg})$ was treated with four doses of $\mathrm{HA}+\mathrm{FA}(0,10,15$, and $20 \mathrm{ml} / \mathrm{L}$ ) to elucidate the effects of $\mathrm{HA}+\mathrm{FA}$ on alleviation of Cd stress, enhancement of plant growth and physiological-biochemical properties of garden cress.

\section{Materials and methods}

Setting up the experiment. Garden cress (Lepidium sativum cv Helen) was grown in polyethylene pots in the greenhouse of Atatürk University, Erzurum. The greenhouse temperature was maintained at an average temperature of $22( \pm 2)^{\circ} \mathrm{C}$ during the day, and $17( \pm 2)^{\circ} \mathrm{C}$ at night.

The soil used in this study was sampled to a depth of $15 \mathrm{~cm}$ from agricultural fields in Erzurum province, Turkey $\left(39^{\circ} 55^{\prime} \mathrm{N}, 41^{\circ} 61^{\prime} \mathrm{E}\right)$. It was dried indoors until it could be crumbled to pass through a $4 \mathrm{~mm}$-sieve and a $2 \mathrm{~mm}$-sieve, for pot experiments and for analyses of physicochemical properties, respectively. The soil is classified as Ustorthents according to the soil taxonomy (Soil Survey Staff 1999). The composition of soil was 33\% sand, $34 \%$ silt, $33 \%$ clay, $1.2 \%$ organic matter. Other physical and chemical properties were: $\mathrm{pH}$ : 5.61 , cation exchange capacity: $15.5 \mathrm{cmol}(+) / \mathrm{kg}$, electrical conductivity: $1.15 \mathrm{dS} / \mathrm{m}$, total $\mathrm{N}: 1.15 \%, \mathrm{P}: 15.5 \mathrm{mg} / \mathrm{kg}, \mathrm{Zn}: 2.30 \mathrm{mg} / \mathrm{kg}$, Cu: $1.1 \mathrm{mg} / \mathrm{kg}$, Fe: $0.5 \mathrm{mg} / \mathrm{kg}$, Mn: $1.8 \mathrm{mg} / \mathrm{kg}$, Cd: $0.05 \mathrm{mg} / \mathrm{kg} \mathrm{Cd}, \mathrm{K}: 2.2 \mathrm{cmol}(+) / \mathrm{kg}, \mathrm{Ca}: 11.0 \mathrm{cmol}(+) / \mathrm{kg}, \mathrm{Mg}$ : $2.25 \mathrm{cmol}(+) / \mathrm{kg}$.

For heavy metal stress treatments, cadmium $\left(\mathrm{CdSO}_{4} \cdot 8 \mathrm{H}_{2} \mathrm{O}\right)$ was mixed with the medium at three different concentrations $(0,100$, and $200 \mathrm{mg} / \mathrm{kg})$ and incubated for 3 weeks.

$\mathrm{HA}+\mathrm{FA}$ was supplied from Humintech GmbH (Grevenbroich / Germany) as a commercial product, namely Powhumus. The content (\% dry wt) of Powhumus was as follows: HA + FA: 80-85\% (Humic acid 90\%, Fulvic acid $10 \%$ ), Potassium as $\mathrm{K}_{2} \mathrm{O}: 10-12 \%$; total organic Nitrogen: $1.0 \%$. Particle size of insoluble constituents was $<100 \mu \mathrm{m}$ and $\mathrm{pH}$ was $9-10$. The element content of Powhumus (\%, mass) was $\mathrm{C}-32, \mathrm{H}-2.8, \mathrm{~N}-1.3$. and the ash content was $22.9 \%$. The element content on ash-free basis (\% wt) was $\mathrm{C}-47.1, \mathrm{H}-3.63, \mathrm{~N}-1.69, \mathrm{O}-47.58$.

To prepare HA + FA (Powhumus) solutions $350 \mathrm{~g}$ Powhumus was dissolved in $1 \mathrm{~L}$ water $(350000 \mathrm{mg} / \mathrm{L})$ and then diluted to obtain three different concentrations $(3500,5250$, and $7000 \mathrm{mg} / \mathrm{L})$. HA + FA solutions were applied to the soil three times a week, starting the day before planting. As a control, $0 \mathrm{mg} / \mathrm{L} \mathrm{HA}+\mathrm{FA}$ was used, instead 150:100:150 kg/ha NPK was mixed into the medium as basal fertilizer.

Seeds of garden cress were sown in pots filled with $1 \mathrm{~L}$ of three-week-incubated garden soil: sand $(1: 1, \mathrm{v}: \mathrm{v})$ mixture in a 1-1.5 cm depth, with 10 seeds per pot. After the seedling emergence, four plants were left in each pot with the same appearance.

Harvest and growth parameters. The pot study was terminated on the 50th day from seed sowing. At harvest, four plants from each sample were taken to measure stem diameter, plant height, leaf number, aerial fresh-dry weight, and root fresh-dry weight. For dry weight measurements, the plant material was kept at $70{ }^{\circ} \mathrm{C}$ for $48 \mathrm{~h}$. To determine the content of proline, sucrose, $\mathrm{MDA}, \mathrm{H}_{2} \mathrm{O}_{2}$, and antioxidant enzyme activity, roughly $20 \mathrm{~g}$ of fresh leaves were frozen in liquid nitrogen and then stored at $-80^{\circ} \mathrm{C}$. Analyses were performed in quadruplicate.

Chlorophyll reading value (CRV as SPAD). The chlorophyll content of the plant leaves was determined by a chlorophyll meter (SPAD_502, Konica Minolta Sensing, Inc., Japan).

Leaf area. The leaf areas of the plants in each application were determined using a leaf area meter (CID-202 Portable Laser Leaf Area Meter, CID Bio-Science, Inc., WA, USA).

Membrane permeability (MP). $\quad$ MP was determined according to Yildirim et al. ${ }^{12}$. 


\begin{tabular}{|c|c|c|c|c|c|}
\hline Cd (mg/kg) & $\mathrm{HA}+\mathrm{FA}(\mathrm{mg} / \mathrm{L})$ & $\begin{array}{l}\text { Leaf fresh weight (g/ } \\
\text { plant) }\end{array}$ & $\begin{array}{l}\text { Leaf dry weight ( } \mathrm{g} / \\
\text { plant) }\end{array}$ & $\begin{array}{l}\text { Root fresh weight (g/ } \\
\text { plant) }\end{array}$ & $\begin{array}{l}\text { Root dry weight (g/ } \\
\text { plant) }\end{array}$ \\
\hline \multirow{4}{*}{0} & 0 & $3.66 \pm 0.09 \mathrm{~b}$ & $0.50 \pm 0.050 \mathrm{~d}$ & $2.63 \pm 0.10 \mathrm{~d}$ & $0.32 \pm 0.012 b$ \\
\hline & 3500 & $4.70 \pm 0.18 \mathrm{a}$ & $0.61 \pm 0.023 b$ & $3.40 \pm 0.07 \mathrm{~b}$ & $0.38 \pm 0.025 a$ \\
\hline & 5250 & $4.91 \pm 0.14 \mathrm{a}$ & $0.55 \pm 0.005 c$ & $3.51 \pm 0.09 \mathrm{a}$ & $0.37 \pm 0.011 \mathrm{a}$ \\
\hline & 7000 & $4.97 \pm 0.16 \mathrm{a}$ & $0.65 \pm 0.017 \mathrm{a}$ & $3.01 \pm 0.12 \mathrm{c}$ & $0.31 \pm 0.018 \mathrm{~b}$ \\
\hline \multirow{4}{*}{100} & 0 & $2.28 \pm 0.04 \mathrm{c}$ & $0.26 \pm 0.014 \mathrm{f}$ & $1.32 \pm 0.01 \mathrm{~g}$ & $0.12 \pm 0.004 \mathrm{~d}$ \\
\hline & 3500 & $2.54 \pm 0.01 \mathrm{c}$ & $0.30 \pm 0.019 \mathrm{e}$ & $1.49 \pm 0.03 \mathrm{f}$ & $0.14 \pm 0.010 \mathrm{c}$ \\
\hline & 5250 & $2.63 \pm 0.03 c$ & $0.30 \pm 0.004 \mathrm{e}$ & $1.63 \pm 0.05 \mathrm{e}$ & $0.13 \pm 0.006 \mathrm{c}$ \\
\hline & 7000 & $2.26 \pm 1.11 \mathrm{c}$ & $0.32 \pm 0.005 \mathrm{e}$ & $1.71 \pm 0.04 \mathrm{e}$ & $0.14 \pm 0.008 c$ \\
\hline \multirow{4}{*}{200} & 0 & $0.43 \pm 0.03 e$ & $0.08 \pm 0.0041$ & $0.14 \pm 0.01 j$ & $0.03 \pm 0.002 \mathrm{~g}$ \\
\hline & 3500 & $1.25 \pm 0.05 \mathrm{~d}$ & $0.13 \pm 0.009 \mathrm{~h}$ & $0.51 \pm 0.031$ & $0.05 \pm 0.004 \mathrm{f}$ \\
\hline & \begin{tabular}{|l|}
5250 \\
\end{tabular} & $1.11 \pm 0.07 \mathrm{~d}$ & $0.14 \pm 0.006 \mathrm{~h}$ & $0.50 \pm 0.031$ & $0.04 \pm 0.001 \mathrm{fg}$ \\
\hline & 7000 & $1.56 \pm 0.05 \mathrm{~d}$ & $0.19 \pm 0.011 \mathrm{~g}$ & $0.91 \pm 0.04 \mathrm{~h}$ & $0.07 \pm 0.004 \mathrm{e}$ \\
\hline
\end{tabular}

Table 1. Effect of cadmium and HA applications on leaf fresh, leaf dry, root fresh and root dry weight of garden cress. The difference between the means indicated by different letters in the same column is statistically significant (Duncan multiple comparison test, $\mathrm{P}<0.05$ ).

Leaf relative water content (LRWC). LRWC was determined according to Yildirim et al. ${ }^{12}$.

$\mathrm{H}_{2} \mathrm{O}_{2}$ and MDA analysis. $\mathrm{H}_{2} \mathrm{O}_{2}$ was determined according to Velikova et al. ${ }^{13}$. The content of $\mathrm{H}_{2} \mathrm{O}_{2}$ was calculated by using a standard calibration curve previously made by using different concentrations of $\mathrm{H}_{2} \mathrm{O}_{2}$. Thiobarbituric acid-reactive substances were measured as MDA, a degraded product of the lipid, which determines the lipid peroxidation. The concentration of MDA was determined from the absorbance curve, by using an extinction coefficient of $155 \mathrm{mmol} \mathrm{L}^{-1} \mathrm{~cm}^{-1}$.

Sucrose and proline analysis. Sucrose concentration was measured by a method given by Chopra et al. ${ }^{14}$. Proline concentration was assayed spectrophotometrically at $520 \mathrm{~nm}^{15}$.

Catalase (CAT), peroxidase (POD) and superoxide dismutase (SOD) activity. CAT, POD, and SOD activities were determined based on the method given by Sahin et al. ${ }^{16}$.

Mineral analysis. Garden cress leaves were ground after being dried at $68^{\circ} \mathrm{C}$ for $48 \mathrm{~h}$ in an oven. Determination of the total $\mathrm{N}$ was achieved by the Kjeldahl method using a Vapodest 10 Rapid Kjeldahl Distillation Unit (Gerhardt, Konigswinter, Germany). An inductively coupled plasma spectrophotometer (Optima 2100 DV, ICP/ OES; Perkin-Elmer, Shelton, CT) was used to determine tissue P, K, Ca, Mg, Fe, Cu, Mn, Zn, B, Cl and $\mathrm{Cd}^{17,18}$.

Statistical analysis. In the experiment, a randomized plot design was used and the obtained data were analyzed using SPSS 20 statistical package program. Data were subjected to variance analysis (ANOVA) and differences of means were determined by Duncan multiple comparison test.

\section{Results}

As can be seen in Table 1, humic + fulvic acid application affected the leaf fresh, leaf dry, root fresh and root dry weights of garden cress significantly under $\mathrm{Cd}$ stress condition. Our results showed that $\mathrm{Cd}$ pollution negatively affected all parameters investigated and the negative effect increased with the increased pollution doses. Without Cd pollution, the highest leaf fresh and leaf dry weight of the plant were obtained when HA + FA was applied at a concentration of $7000 \mathrm{mg} / \mathrm{L}$, whereas the highest root fresh and root dry weight were found when the concentration of HA + FA applied was $3500 \mathrm{mg} / \mathrm{L}$. These findings indicate that, without Cd pollution, leaf fresh weight and dry weight increased $35 \%$ and $30 \%$, respectively with application of $7000 \mathrm{mg} / \mathrm{L} \mathrm{HA}+\mathrm{FA}$, whereas root fresh weight and dry weight increased $33 \%$ and $15 \%$ when $3500 \mathrm{mg} / \mathrm{L}$ HA + FA was applied, compared to the control $(\mathrm{HA}+\mathrm{FA}=0 \mathrm{mg} / \mathrm{L})$.

With Cd pollution, leaf fresh, leaf dry, root fresh and root dry weights were affected negatively. However, the $\mathrm{HA}+\mathrm{FA}$ applications alleviated the deleterious effects of the Cd to the plant. At $200 \mathrm{mg} / \mathrm{kg}$ Cd pollution, HA + FA application at a concentration of $7000 \mathrm{mg} / \mathrm{L}$ increased the leaf fresh, leaf dry, root fresh and root dry weights by $262 \%, 137 \%, 550 \%$ and $133 \%$, respectively, compared to the control (Table 1 ).

The effect of HA + FA applications on the stem diameter, leaf area and CRV of the garden cress under Cd stress is presented in Table 2. Cd pollution significantly decreased the stem diameter, leaf area and CRV in the cress. The most negative effect occurred in $200 \mathrm{mg} / \mathrm{kg}$ Cd pollution. However, HA + FA applications reduced the negative effects of 100 and $200 \mathrm{mg} / \mathrm{kg}$ Cd stress on the cress. At $200 \mathrm{mg} / \mathrm{kg}$ Cd pollution, HA + FA application at a concentration of $7000 \mathrm{mg} / \mathrm{L}$ increased the stem diameter, leaf area and CRV by $92 \%, 104 \%$, and $34 \%$, respectively, compared to the control (Table 2). 


\begin{tabular}{|l|l|l|l|l|l|l|}
\hline $\mathbf{C d}(\mathbf{m g} / \mathbf{k g})$ & HA + FA $(\mathbf{m g} / \mathbf{L})$ & Stem diameter $(\mathbf{m m})$ & Leaf area $\left.\mathbf{( c m}^{2}\right)$ & CRV $($ SPAD) & MP $(\%)$ & LWRC $(\%)$ \\
\hline \multirow{5}{*}{0} & 0 & $3.24 \pm 0.06 \mathrm{~b}$ & $213.30 \pm 4.00 \mathrm{c}$ & $41.00 \pm 1.00 \mathrm{a}$ & $5.93 \pm 0.2 \mathrm{e}$ & $74.39 \pm 1.2 \mathrm{a}$ \\
\cline { 2 - 7 } & 3500 & $3.27 \pm 0.05 \mathrm{~b}$ & $235.93 \pm 4.53 \mathrm{~b}$ & $40.00 \pm 1.73 \mathrm{a}$ & $5.93 \pm 0.3 \mathrm{e}$ & $68.35 \pm 1.4 \mathrm{~cd}$ \\
\cline { 2 - 7 } & 5250 & $3.21 \pm 0.07 \mathrm{~b}$ & $235.21 \pm 6.22 \mathrm{~b}$ & $39.67 \pm 1.15 \mathrm{ab}$ & $5.80 \pm 0.2 \mathrm{e}$ & $70.08 \pm 1.8 \mathrm{bc}$ \\
\cline { 2 - 7 } & 7000 & $3.55 \pm 0.04 \mathrm{a}$ & $236.49 \pm 7.53 \mathrm{~b}$ & $40.67 \pm 0.58 \mathrm{a}$ & $5.66 \pm 0.1 \mathrm{e}$ & $69.33 \pm 1.6 \mathrm{c}$ \\
\hline \multirow{4}{*}{100} & 0 & $2.36 \pm 0.07 \mathrm{e}$ & $139.18 \pm 3.59 \mathrm{e}$ & $35.00 \pm 1.00 \mathrm{~d}$ & $34.06 \pm \mathrm{c} 0.2$ & $54.16 \pm 1.5 \mathrm{~g}$ \\
\cline { 2 - 7 } & 3500 & $2.68 \pm 0.04 \mathrm{c}$ & $204.78 \pm 3.65 \mathrm{~d}$ & $37.67 \pm 0.57 \mathrm{c}$ & $24.96 \pm 0.3 \mathrm{~d}$ & $59.06 \pm 1.7 \mathrm{f}$ \\
\cline { 2 - 7 } & 5250 & $2.57 \pm 0.05 \mathrm{~d}$ & $214.37 \pm 4.92 \mathrm{c}$ & $40.33 \pm 0.58 \mathrm{a}$ & $24.78 \pm 0.4 \mathrm{~d}$ & $64.89 \pm 1.5 \mathrm{de}$ \\
\cline { 2 - 7 } & 7000 & $2.73 \pm 0.04 \mathrm{c}$ & $253.22 \pm 4.99 \mathrm{a}$ & $38.00 \pm 1.01 \mathrm{bc}$ & $23.85 \pm 0.5 \mathrm{~d}$ & $73.87 \pm 1.6 \mathrm{ab}$ \\
\hline \multirow{3}{*}{200} & 0 & $0.85 \pm 0.011$ & $69.75 \pm 4.75 \mathrm{~g}$ & $25.00 \pm 1.10 \mathrm{f}$ & $41.94 \pm 0.8 \mathrm{a}$ & $51.92 \pm 1.8 \mathrm{~g}$ \\
\cline { 2 - 7 } & 3500 & $1.18 \pm 0.03 \mathrm{~h}$ & $100.48 \pm 2.39 \mathrm{f}$ & $31.00 \pm 1.05 \mathrm{e}$ & $35.06 \pm 0.4 \mathrm{bc}$ & $63.64 \pm 1.9 \mathrm{e}$ \\
\cline { 2 - 7 } & 5250 & $1.30 \pm 0.04 \mathrm{~g}$ & $100.65 \pm 5.72 \mathrm{f}$ & $29.67 \pm 1.15 \mathrm{e}$ & $34.91 \pm 0.2 \mathrm{bc}$ & $58.93 \pm 1.3 \mathrm{f}$ \\
\cline { 2 - 7 } & 7000 & $1.64 \pm 0.03 \mathrm{f}$ & $142.40 \pm 2.48 \mathrm{e}$ & $34.33 \pm 0.50 \mathrm{~d}$ & $37.88 \pm 0.3 \mathrm{~b}$ & $68.83 \pm 1.5 \mathrm{~cd}$ \\
\hline
\end{tabular}

Table 2. Effect of cadmium and HA applications on stem diameter, leaf area, CRV, MP and LRWC of garden cress. The difference between the means indicated by different letters in the same column is statistically significant (Duncan multiple comparison test, $\mathrm{P}<0.05$ ).

\begin{tabular}{|c|c|c|c|c|c|}
\hline Cd (mg/kg) & $\mathrm{HA}+\mathrm{FA}(\mathrm{mg} / \mathrm{L})$ & CAT (EU gr/leaf) & POD (EU gr/leaf) & SOD (EU gr/leaf) & \\
\hline \multirow{4}{*}{0} & 0 & $75.15 \pm 3.00 \mathrm{bc}$ & $0.53 \pm 0.01 \mathrm{~g}$ & $81.72 \pm 2.27 \mathrm{gh}$ & \\
\hline & 3500 & $72.38 \pm 2.78 \mathrm{bcd}$ & $0.52 \pm 0.02 \mathrm{~g}$ & $86.86 \pm 3.80 \mathrm{~g}$ & \\
\hline & 5250 & $76.97 \pm 4.04 \mathrm{~b}$ & $0.56 \pm 0.03 \mathrm{~g}$ & $85.19 \pm 3.35 \mathrm{~g}$ & \\
\hline & 7000 & $75.90 \pm 1.27 \mathrm{bc}$ & $0.54 \pm 0.02 \mathrm{~g}$ & $79.57 \pm 1.361$ & \\
\hline \multirow{4}{*}{100} & 0 & $68.05 \pm 1.22 \mathrm{de}$ & $0.58 \pm 0.01 \mathrm{~g}$ & $131.76 \pm 2.42 \mathrm{~d}$ & \\
\hline & 3500 & $71.39 \pm 1.64 \mathrm{~cd}$ & $1.16 \pm 0.04 \mathrm{e}$ & $128.58 \pm 2.48 \mathrm{de}$ & \\
\hline & 5250 & $73.69 \pm 1.36 \mathrm{bc}$ & $1.61 \pm 0.09 \mathrm{~d}$ & $122.33 \pm 2.85 \mathrm{f}$ & \\
\hline & 7000 & $56.92 \pm 2.32 f$ & $1.76 \pm 0.06 \mathrm{c}$ & $126.07 \pm 1.81 \mathrm{ef}$ & \\
\hline \multirow{4}{*}{200} & 0 & $91.29 \pm 1.97 \mathrm{a}$ & $0.78 \pm 0.05 f$ & $168.57 \pm 3.65 \mathrm{a}$ & \\
\hline & 3500 & $77.28 \pm 6.19 \mathrm{~b}$ & $1.84 \pm 0.03 \mathrm{c}$ & $156.28 \pm 4.55 \mathrm{~b}$ & \\
\hline & 5250 & $65.25 \pm 1.05 \mathrm{e}$ & $2.01 \pm 0.05 \mathrm{~b}$ & $137.59 \pm 1.99 \mathrm{c}$ & \\
\hline & 7000 & $51.58 \pm 3.22 \mathrm{~g}$ & $2.23 \pm 0.13 \mathrm{a}$ & $132.75 \pm 2.68 \mathrm{~cd}$ & \\
\hline $\mathrm{Cd}(\mathrm{mg} / \mathrm{kg})$ & $\mathrm{HA}+\mathrm{FA}(\mathrm{mg} / \mathrm{L})$ & $\mathrm{H}_{2} \mathrm{O}_{2}(\mathrm{mmol} / \mathrm{kg})$ & MDA (nmol/g) & Proline $(\mu \mathrm{g} / \mathrm{g})$ & Sucrose (\%) \\
\hline \multirow{4}{*}{0} & 0 & $140.67 \pm 2.08 \mathrm{f}$ & $4.34 \pm 0.19 \mathrm{de}$ & $55.33 \pm 2.84 \mathrm{fg}$ & $41.42 \pm 1.99 \mathrm{~cd}$ \\
\hline & 3500 & $135.00 \pm 10.00 \mathrm{f}$ & $4.57 \pm 0.16 \mathrm{cde}$ & $55.39 \pm 1.98 \mathrm{fg}$ & $48.49 \pm 2.02 \mathrm{~b}$ \\
\hline & 5250 & $138.88 \pm 3.40 \mathrm{f}$ & $4.65 \pm 0.09$ cde & $55.90 \pm 4.39 \mathrm{fg}$ & $39.40 \pm 2.46 \mathrm{de}$ \\
\hline & 7000 & $136.33 \pm 4.04 \mathrm{f}$ & $3.16 \pm 0.18 \mathrm{f}$ & $52.00 \pm 1.08 \mathrm{~g}$ & $28.65 \pm 2.90 \mathrm{~g}$ \\
\hline \multirow{4}{*}{100} & 0 & $467.78 \pm 18.93 b$ & $9.82 \pm 1.26 \mathrm{~b}$ & $126.07 \pm 2.07 \mathrm{~b}$ & $44.46 \pm 1.38 \mathrm{bc}$ \\
\hline & 3500 & $215.03 \pm 7.58 \mathrm{e}$ & $5.28 \pm 0.26 c$ & $96.94 \pm 0.51 \mathrm{c}$ & $33.89 \pm 2.93 \mathrm{f}$ \\
\hline & 5250 & $143.90 \pm 3.53 \mathrm{f}$ & $3.92 \pm 0.24 \mathrm{ef}$ & $79.55 \pm 2.8 \mathrm{~d}$ & $45.14 \pm 2.09 \mathrm{bc}$ \\
\hline & 7000 & $116.72 \pm 2.53 \mathrm{~g}$ & $3.48 \pm 0.15 f$ & $31.04 \pm 1.38 \mathrm{~h}$ & $22.87 \pm 3.15 \mathrm{~h}$ \\
\hline \multirow{4}{*}{200} & 0 & $662.68 \pm 8.93 a$ & $13.67 \pm 0.92 \mathrm{a}$ & $200.37 \pm 7.13 \mathrm{a}$ & $62.95 \pm 0.32 \mathrm{a}$ \\
\hline & 3500 & $311.67 \pm 9.24 \mathrm{c}$ & $5.34 \pm 0.13 c$ & $82.39 \pm 4.12 \mathrm{~d}$ & $36.77 \pm 0.68 \mathrm{ef}$ \\
\hline & 5250 & $246.25 \pm 2.67 \mathrm{~d}$ & $5.11 \pm 0.09 \mathrm{~cd}$ & $72.89 \pm 3.48 \mathrm{e}$ & $25.92 \pm 1.08 \mathrm{gh}$ \\
\hline & 7000 & $218.94 \pm 2.82 \mathrm{e}$ & $4.37 \pm 0.06 \mathrm{de}$ & $59.33 \pm 2.72 f$ & $27.60 \pm 4.99 \mathrm{~g}$ \\
\hline
\end{tabular}

Table 3. Effect of cadmium and HA applications on CAT, POD, SOD, $\mathrm{H}_{2} \mathrm{O}_{2}$, MDA, proline and sucrose activities of garden cress. The difference between the means indicated by different letters in the same column is statistically significant (Duncan multiple comparison test, $\mathrm{P}<0.05$ ).

The present study showed that Cd pollution increased the MP value in the cress while decreasing the LRWC value. The highest MP and the lowest LRWC values were determined in plants treated with $200 \mathrm{mg} / \mathrm{kg} \mathrm{Cd}$. The $\mathrm{HA}+\mathrm{FA}$ applications mitigated the adverse effects of $\mathrm{Cd}$ and at $200 \mathrm{mg} / \mathrm{kg} \mathrm{Cd}$ pollution, HA + FA application at a concentration of $7000 \mathrm{mg} / \mathrm{L}$ increased the MP and LRWC values by $537 \%$ and $32 \%$, respectively, compared to the control (Table 2).

CAT, POD, and SOD activities were found to increase with increasing Cd pollution. At $200 \mathrm{mg} / \mathrm{kg}$ Cd pollution, $7000 \mathrm{mg} / \mathrm{L} \mathrm{HA}+\mathrm{FA}$ application decreased CAT and SOD activities by $43 \%$ and $21 \%$ respectively, and increased POD activity by $186 \%$, compared to the samples without HA + FA application (Table 3 ). Although the highest $\mathrm{H}_{2} \mathrm{O}_{2}$, MDA, proline and sucrose values were obtained at $200 \mathrm{mg} / \mathrm{kg}$ Cd pollution, $\mathrm{HA}+\mathrm{FA}$ application at 


\begin{tabular}{|l|l|l|l|l|l|l|}
\hline $\mathbf{C d}(\mathbf{m g} / \mathbf{k g})$ & $\mathbf{H A}+\mathbf{F A}(\mathbf{m g} / \mathbf{L})$ & $\mathbf{N}(\%)$ & $\mathbf{P}(\mathbf{m g} / \mathbf{k g})$ & $\mathbf{K}(\mathbf{m g} / \mathbf{k g})$ & $\mathbf{C a}(\mathbf{m g} / \mathbf{k g})$ & $\mathbf{M g}(\mathbf{m g} / \mathbf{k g}$ \\
\hline \multirow{4}{*}{0} & 0 & $1.93 \pm 0.04 \mathrm{e}$ & $1931 \pm 41.13 \mathrm{~d}$ & $7164 \pm 108.70 \mathrm{ef}$ & $5076 \pm 184.53 \mathrm{de}$ & $3724 \pm 85.13 \mathrm{~d}$ \\
\cline { 2 - 7 } & 3500 & $2.06 \pm 0.02 \mathrm{~d}$ & $2055 \pm 40.08 \mathrm{c}$ & $7596 \pm 73.63 \mathrm{e}$ & $5536 \pm 58.02 \mathrm{c}$ & $3973 \pm 60.75 \mathrm{c}$ \\
\cline { 2 - 7 } & 5250 & $2.26 \pm 0.03 \mathrm{c}$ & $2289 \pm 42.92 \mathrm{~b}$ & $10,374 \pm 415.07 \mathrm{~b}$ & $5276 \pm 115.41 \mathrm{~d}$ & $4120 \pm 55.3 \mathrm{~b}$ \\
\cline { 2 - 7 } & 7000 & $2.56 \pm 0.05 \mathrm{a}$ & $2446 \pm 37.93 \mathrm{a}$ & $13,046 \pm 85.40 \mathrm{a}$ & $6980 \pm 181.85 \mathrm{a}$ & $4570 \pm 47.80 \mathrm{a}$ \\
\hline \multirow{3}{*}{100} & 0 & $1.21 \pm 0.03 \mathrm{~h}$ & $1405 \pm 40.61 \mathrm{~g}$ & $5010 \pm 168.27 \mathrm{~h}$ & $4393 \pm 124.57 \mathrm{f}$ & $2560 \pm 53.70 \mathrm{~h}$ \\
\cline { 2 - 7 } & 3500 & $1.33 \pm 0.01 \mathrm{~g}$ & $1711 \pm 17.61 \mathrm{e}$ & $7419 \pm 127.60 \mathrm{e}$ & $4885 \pm 119.71 \mathrm{e}$ & $2655 \pm 67.54 \mathrm{~g}$ \\
\cline { 2 - 7 } & 5250 & $2.22 \pm 0.08 \mathrm{c}$ & $1561 \pm 49.34 \mathrm{f}$ & $8132 \pm 111.87 \mathrm{~d}$ & $5694 \pm 175.13 \mathrm{c}$ & $2970 \pm 75.21 \mathrm{f}$ \\
\cline { 2 - 7 } & 7000 & $2.36 \pm 0.07 \mathrm{~b}$ & $1521 \pm 19.70 \mathrm{f}$ & $9009 \pm 190.06 \mathrm{c}$ & $6189 \pm 289.64 \mathrm{~b}$ & $3100 \pm 47.66 \mathrm{e}$ \\
\hline \multirow{3}{*}{200} & 0 & $1.17 \pm 0.01 \mathrm{~h}$ & $1064 \pm 58.88 \mathrm{j}$ & $3912 \pm 63.991$ & $2709 \pm 34.20 \mathrm{~h}$ & $1920 \pm 57.81 \mathrm{k}$ \\
\cline { 2 - 7 } & 3500 & $1.76 \pm 0.03 \mathrm{f}$ & $1265 \pm 27.381$ & $6217 \pm 371.25 \mathrm{~g}$ & $3654 \pm 160.33 \mathrm{~g}$ & $2320 \pm 49.45 \mathrm{j}$ \\
\cline { 2 - 7 } & 5250 & $1.92 \pm 0.05 \mathrm{e}$ & $1343 \pm 37.88 \mathrm{gh}$ & $6835 \pm 606.48 \mathrm{f}$ & $3603 \pm 22.65 \mathrm{~g}$ & $2580 \pm 58.66 \mathrm{i}$ \\
\cline { 2 - 7 } & 7000 & $2.05 \pm 0.08 \mathrm{~d}$ & $1313 \pm 17.67 \mathrm{~h} 1$ & $7209 \pm 92.88 \mathrm{ef}$ & $5068 \pm 163.03 \mathrm{de}$ & $2690 \pm 39.40 \mathrm{~h}$ \\
\hline
\end{tabular}

Table 4. Effect of cadmium and HA applications on macro element content of garden cress. The difference between the means indicated by different letters in the same column is statistically significant (Duncan multiple comparison test, $\mathrm{P}<0.05)$.

\begin{tabular}{|c|c|c|c|c|c|}
\hline $\mathrm{Cd}(\mathrm{mg} / \mathrm{kg})$ & $\mathrm{HA}+\mathrm{FA}(\mathrm{mg} / \mathrm{L})$ & $\mathrm{Fe}(\mathrm{mg} / \mathrm{kg})$ & $\mathrm{Cu}(\mathrm{mg} / \mathrm{kg})$ & Mn (mg/kg) & Zn (mg/kg) \\
\hline \multirow{4}{*}{0} & 0 & $97.75 \pm 5.42 \mathrm{~d}$ & $9.93 \pm 0.31 \mathrm{~d}$ & $21.44 \pm 1.05 \mathrm{~b}$ & $9.33 \pm 0.35 c$ \\
\hline & 3500 & $101.21 \pm 4.90 \mathrm{~cd}$ & $11.61 \pm 0.64 \mathrm{c}$ & $21.80 \pm 0.73 \mathrm{~b}$ & $10.18 \pm 0.57 \mathrm{bc}$ \\
\hline & 5250 & $148.77 \pm 5.60 \mathrm{~b}$ & $15.26 \pm 0.50 \mathrm{~b}$ & $21.38 \pm 0.48 \mathrm{~b}$ & $10.29 \pm 0.47 \mathrm{bc}$ \\
\hline & 7000 & $174.35 \pm 8.72 \mathrm{a}$ & $25.50 \pm 0.69 a$ & $20.47 \pm 2.30 \mathrm{bc}$ & $10.83 \pm 0.52 b$ \\
\hline \multirow{4}{*}{100} & 0 & $59.20 \pm 0.94 \mathrm{~h}$ & $5.82 \pm 0.38 \mathrm{f}$ & $18.19 \pm 1.65 \mathrm{de}$ & $5.86 \pm 0.38 \mathrm{e}$ \\
\hline & 3500 & $85.82 \pm 5.57 f$ & $8.33 \pm 0.22 \mathrm{e}$ & $19.67 \pm 0.48 \mathrm{bcd}$ & $6.61 \pm 0.33 \mathrm{de}$ \\
\hline & 5250 & $100.13 \pm 4.26 \mathrm{~cd}$ & $10.27 \pm 0.70 \mathrm{~cd}$ & $24.41 \pm 1.16 \mathrm{a}$ & $9.94 \pm 1.74 \mathrm{bc}$ \\
\hline & 7000 & $107.80 \pm 4.85 c$ & $14.11 \pm 0.77 \mathrm{~b}$ & $16.91 \pm 0.93 \mathrm{ef}$ & $12.05 \pm 0.62 \mathrm{a}$ \\
\hline \multirow{4}{*}{200} & 0 & $50.87 \pm 1.221$ & $3.24 \pm 0.14 \mathrm{~g}$ & $16.90 \pm 0.23 \mathrm{ef}$ & $6.48 \pm 0.39 \mathrm{de}$ \\
\hline & 3500 & $73.88 \pm 1.76 \mathrm{~g}$ & $5.47 \pm 0.44 \mathrm{f}$ & $18.80 \pm 1.58 \mathrm{cde}$ & $7.68 \pm 0.47 \mathrm{~d}$ \\
\hline & 5250 & $86.81 \pm 2.49 \mathrm{ef}$ & $5.09 \pm 0.10 \mathrm{f}$ & $15.88 \pm 0.99 f$ & $9.92 \pm 0.66 \mathrm{bc}$ \\
\hline & 7000 & $94.22 \pm 2.77 \mathrm{de}$ & $7.90 \pm 0.01 \mathrm{e}$ & $16.93 \pm 0.14 \mathrm{ef}$ & $10.72 \pm 0.62 b$ \\
\hline $\mathrm{Cd}(\mathrm{mg} / \mathrm{kg})$ & $\mathrm{HA}+\mathrm{FA}(\mathrm{mg} / \mathrm{L})$ & B (mg/kg) & Cd (mg/kg) & $\mathrm{Cl}(\mathrm{mg} / \mathrm{kg})$ & \\
\hline \multirow{4}{*}{0} & 0 & $7.31 \pm 0.57 \mathrm{e}$ & $0.20 \pm 0.02 \mathrm{~g}$ & $0.32 \pm 0.02 \mathrm{ef}$ & \\
\hline & 3500 & $8.04 \pm 0.46 \mathrm{de}$ & $0.25 \pm 0.01 \mathrm{~g}$ & $0.33 \pm 0.01 \mathrm{ef}$ & \\
\hline & 5250 & $10.16 \pm 0.61 \mathrm{~b}$ & $0.32 \pm 0.02 \mathrm{~g}$ & $0.31 \pm 0.01$ ef & \\
\hline & 7000 & $11.99 \pm 0.70 \mathrm{a}$ & $0.34 \pm 0.03 \mathrm{~g}$ & $0.28 \pm 0.02 \mathrm{f}$ & \\
\hline \multirow{4}{*}{100} & 0 & $4.76 \pm 0.21 \mathrm{~g}$ & $43.01 \pm 0.20 \mathrm{c}$ & $0.51 \pm 0.05 \mathrm{~b}$ & \\
\hline & 3500 & $6.36 \pm 0.55 f$ & $25.99 \pm 0.19 \mathrm{~d}$ & $0.40 \pm 0.02 \mathrm{~cd}$ & \\
\hline & 5250 & $8.77 \pm 0.37 c d$ & $7.92 \pm 1.34 \mathrm{e}$ & $0.46 \pm 0.01 b c$ & \\
\hline & 7000 & $10.01 \pm 0.58 \mathrm{~b}$ & $1.12 \pm 0.81 \mathrm{~d}$ & $0.43 \pm 0.05 c d$ & \\
\hline \multirow{4}{*}{200} & 0 & $4.12 \pm 0.20 \mathrm{~g}$ & $96.61 \pm 0.26 \mathrm{a}$ & $1.02 \pm 0.08 \mathrm{a}$ & \\
\hline & 3500 & $7.36 \pm 0.15 \mathrm{e}$ & $47.05 \pm 0.98 \mathrm{~b}$ & $0.36 \pm 0.04 \mathrm{de}$ & \\
\hline & 5250 & $7.47 \pm 0.29 \mathrm{e}$ & $13.98 \pm 1.44 \mathrm{e}$ & $0.20 \pm 0.02 \mathrm{~g}$ & \\
\hline & 7000 & $8.87 \pm 0.10 c$ & $3.00 \pm 1.13 \mathrm{f}$ & $0.20 \pm 0.04 \mathrm{~g}$ & \\
\hline
\end{tabular}

Table 5. Effect of cadmium and HA applications on micro element content of garden cress. The difference between the means indicated by different letters in the same column is statistically significant (Duncan multiple comparison test, $\mathrm{P}<0.05)$.

a concentration of $7000 \mathrm{mg} / \mathrm{L}$ successfully alleviated the deleterious effects of Cd stress by decreasing $\mathrm{H}_{2} \mathrm{O}_{2}, \mathrm{MDA}_{\text {, }}$ proline and sucrose values by $66 \%, 68 \%, 70 \%$ and $56 \%$, respectively at $200 \mathrm{mg} / \mathrm{kg}$ Cd pollution level (Table 3).

Cd pollution decreased all mineral elements in garden cress investigated within this work, but for the cadmium itself. On the other hand, application of HA + FA, enhanced the mineral element content of the garden cress except for $\mathrm{Cd}$ (Tables 4, 5). The highest N, P, K, Ca, Mg, Fe, Mn, Cu, Mn, Zn, B, and Cl were obtained from $7000 \mathrm{mg} / \mathrm{L} \mathrm{HA}+\mathrm{FA}$ application without Cd pollution, while the lowest values were determined from $0 \mathrm{ml}$ $\mathrm{HA}+\mathrm{FA}$ with $200 \mathrm{mg} / \mathrm{kg}$ Cd pollution treatment. HA + FA application at a concentration of $7000 \mathrm{mg} / \mathrm{L}$ successfully alleviated the deleterious effects of Cd stress by increased $\mathrm{N}, \mathrm{P}, \mathrm{K}, \mathrm{Ca}, \mathrm{Mg}, \mathrm{Fe}, \mathrm{Mn}, \mathrm{Cu}, \mathrm{Mn}, \mathrm{Zn}$, and B by $75 \%, 23 \%, 84 \%, 87 \%, 40 \%, 85 \%, 143 \%, 1 \%, 65 \%$, and $115 \%$, respectively. On the other hand, HA + FA application 
at a concentration of $7000 \mathrm{mg} / \mathrm{L}$ successfully reduced $\mathrm{Cd}$ and $\mathrm{Cl}$ uptake, by $95 \%$ and $80 \%$, respectively (Tables 4 , $5)$.

\section{Discussion}

Heavy metals often cause toxic effects on plants such as chlorosis, inhibition of growth and photosynthesis, water balance variations and nutrient assimilation, which ultimately lead to plant death ${ }^{19}$. Heavy metals exhibit toxicity through four suggested mechanisms in plants. These are: (i) competition with nutrient cations for adsorption at the root surface (e.g. competition of As and Cd with P and Zn, respectively, for adsorption); (ii) inactivation of sulfhydryl groups (- $\mathrm{SH}$ ) by direct interaction, which disrupts plant's structure and function; (iii) collapsing the function of enzymes by displacing essential cations from specific binding sites; and (iv) the production of active oxygen species (ROS), which ultimately damage macromolecules ${ }^{20}$.

$\mathrm{Cd}$ is considered an important pollutant because of its high toxicity and high water solubility. Cadmium can alter mineral uptake through its effects on the presence of minerals in the soil or through a reduction in the population of soil microbes. Cadmium may have a negative effect on stomatal conductivity, perspiration, and photosynthesis efficiency. Chlorosis, leaf folds, and scrub are the common and easy-to-see symptoms of cadmium toxicity of plants. Cd also decrease nitrate uptake and its transport from the roots to the shoots by inhibiting nitrate reductase activity ${ }^{21}$.

In this study, Cd stress was found to affect the growth of garden cress negatively (Table 1). While the LRWC decreased in the cress grown under Cd stress, the MP increased (Table 2). Similarly, previous studies showed the negative effects of Cd stress on plant growth characteristics in radish ${ }^{22}$, garden $\operatorname{cress}^{23,24}$ and lettuce ${ }^{25}$.

On the other hand, HA + FA applications in cress grown under Cd stress positively affected the plant growth (Table 1). Furthermore, HA + FA increased LRWC and decreased MP (Table 2). Similarly, it was reported in previous studies that HA + FA applications mitigated the negative impact of heavy metal stress on plant growth properties in lettuce ${ }^{26}$ (Haghighi et al. 2010), radish ${ }^{22}$, curly lettuce ${ }^{9}$, triticale ${ }^{27}$, corn $^{28}$ and wheat ${ }^{29}$. Humic substances are natural organic polyelectrolytes found in humus, which stabilize organic matter in the soil. Many authors have reported the ability of humic substances to increase the growth of different plant species that grow under different stress conditions ${ }^{27}$. HA + FA positively affect plant growth and yield, directly or indirectly, by improving some physical and chemical properties of the soil. Moreover, HA + FA changes the solubility and bioavailability of toxic heavy metals by forming compounds with them through strong bonds which in turn reduce the heavy metal stress on plant growth ${ }^{9}$. These strong bonds formed with heavy metals are a result of cation exchange capacities (CEC) of humic and fulvic acids. Owing to the high number of carboxyl and phenolic hydroxyl groups in their structure, humic substances have high cation exchange capacities (CEC), which are $600-890 \mathrm{cmol}(+) / \mathrm{kg}$ and $1000-1230 \mathrm{cmol}(+) / \mathrm{kg}$ for humic acid and fulvic acid, respectively $\mathrm{y}^{30,31}$. These capacities are 5 to 100 times higher than that of common clay minerals, which makes humic substances perfect stabilizing agents. In addition, although there are various methods and techniques that have been used for stabilization of $\mathrm{Cd}, \mathrm{Pb}, \mathrm{Cu}$, and $\mathrm{Zn}$, application of humic substances is the only non-chemical and natural method.

In this work, in the absence of Cd stress, no change in the CAT activity was observed due to the application of HA + FA. On the other hand, the effect of Cd stress on CAT activity was found to vary with the concentration of $\mathrm{Cd}$ applied for control samples $(\mathrm{HA}+\mathrm{FA}=0)$, i.e. decreased when the Cd pollution level was $100 \mathrm{mg} /$ $\mathrm{kg}$, however increased once the Cd concentration was changed to $200 \mathrm{mg} / \mathrm{kg}$. Although CAT activity reached a very high level in $200 \mathrm{mg} / \mathrm{kg} \mathrm{Cd}$ polluted control sample (around $90 \mathrm{EU} / \mathrm{gr}$ leaf), application of HA + FA at a concentration of $7000 \mathrm{mg} / \mathrm{L}$ decreased CAT activity significantly, i.e. to the levels (around $50 \mathrm{EU} / \mathrm{gr}$ leaf) even lower than when no Cd stress was present (around 75 EU/gr leaf) (Table 3).

Our results also showed that, Cd stress conditions increased POD and SOD activity in garden cress. POD activity was increased even more with HA + FA applications whereas and SOD activity was decreased with applications of HA + FA (Table 3 ).

It has been shown in different studies that various environmental stresses (salinity, water deficit stress and heavy metal stress) affect the enzyme activity ${ }^{32}$. Shao et al. ${ }^{33}$ showed that antioxidant activity enhanced under stress conditions. On the other hand, Sergiev et al. ${ }^{27}$ suggested that heavy metal applications did not have a significant effect on CAT activity, whereas it increased SOD, GST (glutathione-S-transferase), and GPOX (guaiacol peroxidase) activity in triticale. Similar to our results, Ozkay et al. ${ }^{9}$ indicated that heavy metal stress increased SOD activity in curly lettuce, and HA applications supported this increase. The researchers reported that HA applications led to a further increase in SOD and GST activity, which served as a scavenger of reactive oxygen species. This positive effect of HA may most likely be due to the possibility of forming chelating complexes with HA in the nutrient medium or in the plant. Studies showed that humic acid and fulvic acid efficiently immobilize heavy metals and to a larger extent in the mineral soil ${ }^{34}$. Retention capacity was directly related to the amount of added fulvic acid content in the humic substance. The mobility of fulvic acid (FA) is higher due to its smaller size (molecular weight) and higher oxygen content which is twice of HA. In addition, FA is much more reactive because of the presence of many carboxyl $(\mathrm{COOH})$ and hydroxyl $(\mathrm{COH})$ groups (ranges from 520 to $1120 \mathrm{cmol}$ $(\mathrm{H}+) / \mathrm{kg})$ in its structure, which makes the exchange capacity of FA more than double that of HA.

The behavior of metals in the soil was affected by humic and fulvic acid application. The effects of HA and FA on $\mathrm{Pb}, \mathrm{Cd}, \mathrm{Ni}$ in soil and availability to plants have been extensively investigated. But, inconsistent findings have been reported due to the complex nature of HA and FA and substantial differences in soil characteristics ${ }^{35-37}$.

Earlier reports have indicated that humic and fulvic acid treatments affected plants by affecting the exchangeable nutrient forms in the soil ${ }^{35}$ and decreased $\mathrm{Pb}$, and $\mathrm{Cd}$ accumulation ${ }^{38}$. They suggested, humic and fulvic acid can be employed to immobilize $\mathrm{Pb}$ and $\mathrm{Cd}$ in soil. Studies conducted generally focused on the effects of humic acid, while leaving the effects of fulvic acid in humic-made content unevaluated. Previous studies reported the inhibitory effects of humic substances on heavy metal in acidic soils ${ }^{39-43}$. On the contrary, in alkaline soils, 
humic substances were found to stimulate the metal availability ${ }^{36,37}$. This contradiction can be explained by the unnoticed effect of fulvic acid in the humic content. The underlying reason why the prohibitive impacts of humic substances on metal availability were found at low $\mathrm{pH}$ is that the bond formation between metals and fulvic acid dominated under acidic conditions. In fact, in cases where the fulvic acid content in humic compounds is 5\% or more, the capacity of heavy metal binding and fixing is particularly high in neutral and light acid soils. On the contrary, humic bits of humic acid content, which is very high compared to fulvic acid content, increases the availability of heavy metal. Thus, it can be said that humic acids are effective for heavy metal bioremediation because humic acids can interrelate with metals to form metal-humic complexes ${ }^{34}$. On the other hand, fulvic acids have been reported to inhibit metal availability and might be employed to decrease metal accumulation in the polluted acidic soils. Humic acid had a stimulating impact on heavy metal presence and were very good for metal bioremediation in alkaline soils ${ }^{36,37}$.

As a result, Cd pollution negatively affected plant growth parameters such as plant fresh and dry weight. However, HA + FA applications have been found to reduce this negative effect Considering the plant growth parameters, the best results were determined when HA + FA concentration was $7000 \mathrm{mg} / \mathrm{L}$. We have shown that, it is critical to apply a humic substance with high percentage of FA, which was $10 \%$ in this study, to mitigate the adverse effects of heavy metal stress on plant growth.

Received: 19 November 2020; Accepted: 17 March 2021

Published online: 13 April 2021

\section{References}

1. Achakzai, A. K. K., Bazai, Z. A. \& Kayani, S. A. Accumulation of heavy metals by lettuce (Lactuca satvva L.) Irrigated with different levels of wastewater of quetta city. Pak. J. Bot. 43(6), 2953-2960 (2011).

2. Masindi, V. \& Muedi, K.L. Environmental Contamination by Heavy Metals, Heavy Metals, Hosam El-Din M. Saleh and Refaat F. Aglan, IntechOpen. https://doi.org/10.5772/intechopen.76082. https://www.intechopen.com/books/heavy-metals/environmen tal-contamination-by-heavy-metals (2018).

3. Medyńska-Juraszek, A., Rivier, P. A., Rasse, D. \& Joner, E. J. Biochar affects heavy metal uptake in plants through interactions in the Rhizosphere. Appl. Sci. 10, 5105. https://doi.org/10.3390/app10155105 (2020).

4. Memon, A. R. \& Schröder, P. Implications of metal accumulation mechanisms to phytoremediation. Environ. Sci. Pollut. Res. Int. 16(2), 162-175 (2009).

5. Liu, M. et al. Insight into the cadmium and zinc binding potential of humic acids derived from composts by EEM spectra combined with PARAFAC analysis. Open Chem. 18, 58-68 (2020).

6. Monteiro, M. S., Santos, C., Soares, A. M. V. M. \& Mann, R. M. Assessment of biomarkers of cadmium stres in lettuce. Ecotoxicol. Environ. Saf. 72, 811-818. https://doi.org/10.1016/j.ecoenv.2008.08.002 (2009).

7. Ismael, M. et al. Cadmium in plants: Uptake, toxicity, and its interactions with selenium fertilizers. Metallomics 11, 255-277. https://doi.org/10.1039/C8MT00247A (2019).

8. Dobrikova, A. G. et al. Cadmium toxicity in Salvia sclarea L.: An integrative response of element uptake, oxidative stress markers, leaf structure and photosynthesis. Ecotoxicol. Environ. Saf. 209, 111851. https://doi.org/10.1016/j.ecoenv.2020.111851 (2021).

9. Ozkay, F., Kıran, S., Kuşvuran, S. \& Ellialtıoglu, S. S. Hümik asit uygulamasının kıvırcık salata bitkisinde ağır metal stresi zararını azaltma etkisi. Turk. J. Agric. Food Sci. Technol. 4(6), 431-437. https://doi.org/10.24925/turjaf.v4i6.431-437.542 (2016).

10. Alexander, P. D., Alloway, B. J. \& Dourado, A. M. Genotypic variations in the accumulation of $\mathrm{Cd}, \mathrm{Cu}, \mathrm{Pb}$ and $\mathrm{Zn}$ exhibited by six commonly grown vegetables. Environ. Pollut. 144(3), 736-745. https://doi.org/10.1016/j.envpol.2006.03.001 (2006).

11. Mukhopadhyay, D., Parihar, S. S., Chauhan, J. S. \& Preeti, S. C. J. Effect of temperature and desiccation on seed viability of Lepidium sativum L. N. Y. Sci. J. 3(5), 34-36 (2010).

12. Yildirim, E. et al. Roles of glycine betaine in mitigating deleterious effect of salt stress on lettuce (Lactuca sativa L.). Arch. Agron. Soil Sci. 61(12), 1673-1689. https://doi.org/10.1080/03650340.2015.1030611 (2015).

13. Velikova, V., Yordanov, I. \& Edreva, A. Oxidative stress and some antioxidant systems in acid rain-treated bean plants: Protective role of exogenous polyamines. Plant Sci. 151(1), 59-66 (2000).

14. Chopra, J., Kaur, N. \& Gupta, A. K. Ontogenic changes in enzymes of carbon metabolism in relation to carbohydrate status in developing mungbean reproductive structures. Phytochem. 53(5), 539-548. https://doi.org/10.1016/S0031-9422(99)00545-2 (2000).

15. Dadasoglu, E. et al. Nitric oxide enhances salt tolerance through regulating antioxidant enzyme activity and nutrient uptake in pea. Legume Res. 44(1), 41-45. https://doi.org/10.18805/LR-540 (2021).

16. Sahin, U. et al. Effects of individual and combined effects of salinity and drought on physiological, nutritional and biochemical properties of cabbage (Brassica oleracea var. capitata). Sci. Hortic. 240, 196-204. https://doi.org/10.1016/j.scienta.2018.06.016 (2018).

17. Mertens, D. AOAC Official Method 922.02. Plants preparation of laboratuary sample. Official Methods of Analysis, 18th edn. Horwitz, W., and G.W. Latimer, (Eds). Chapter 3, AOAC-International Suite 500, 481. North Frederick Avenue, Gaitherburg, Maryland, USA. pp. 1-2 (2005a).

18. Mertens, D. AOAC Official Method 975.03. Metal in plants and pet foods. Official Methods of Analysis, 18th edn. Horwitz, W., and G.W. Latimer, (Eds). Chapter 3, AOACInternational Suite 500, 481. North Frederick Avenue, Gaitherburg, Maryland, USA. pp. 3-4 (2005).

19. Singh, S., Parihar, P., Singh, R., Singh, V. P. \& Prasad, S. M. Heavy metal tolerance in plants: Role of transcriptomics, proteomics, metabolomics, and ionomics. Front. Plant Sci. 6, 1143. https://doi.org/10.3389/fpls.2015.01143 (2016).

20. Sharma, S. S. \& Dietz, K. J. The relationship between metal toxicity and cellular redox imbalance. Trends Plant Sci. 14, 43-50. https://doi.org/10.1016/j.tplants.2008.10.007 (2009).

21. Prerna, S., Indra, S. \& Kavita, S. Reduced activity of nitrate reductase under heavy metal cadmium stress in rice: An in silico answer. Front. Plant Sci. 9, 1948. https://doi.org/10.3389/fpls.2018.01948 (2019).

22. Farouk, S., Mosa, A. A., Taha, A. A., Ibrahim, H. M. \& EL-Gahmery, A. M. Protective effect of humic acid and chitosan on radish (Raphanus sativus, L. var. sativus) plants subjected to cadmium stress. J. Stress Physiol. Biochem. 7(2), 99-116 (2011).

23. Pavel, V. L., Sobariu, D. L., Diaconu, M., Stătescu, F. \& Gavrilescu, M. Effects of heavy metals on Lepidium sativum germination and growth. Environ. Eng. Manag. J. 12(4), 727-733. https://doi.org/10.2478/v10032-010-0005-Z (2013).

24. Pirdosti, K. M., Movahedi, Z. \& Rostami, M. Effect of cadmium stress on morpho-physiological traits in garden cress and radish in an aeroponic system. Iran. J. Plant Physiol. 9(1), 2591-2599. https://doi.org/10.22034/IJPP.2018.545615 (2018).

25. Loi, N. N., Sanzharova, N. U., Shchagina, N. I. \& Mironova, M. P. The effect of cadmium toxicity on the development of lettuce plants on contaminated sod-podzolic soil. Russ. Agric. Sci. 44(1), 49-52. https://doi.org/10.3103/S1068367418010111 (2017).

26. Haghighi, M., Kafi, M., Fang, P. \& Gui-Xiao, L. Humic acid decreased hazardous of cadmium toxicity on lettuce (Lactuca sativa L.). Veg. Crops Res. Bull. 72, 49-61. https://doi.org/10.2478/v10032-010-0005-z (2010). 
27. Sergiev, I. et al. Protective effect of humic acids against heavy metal stress in triticale. Comptes Rendus Acad. Bulgare Sci. Tome. 66(1), 53-59 (2012).

28. Chaab, A., Moezzi, A., Sayyad, G. \& Chorom, M. Effect of chromium and cadmium on growth parameters and biochemical responses in soil treated with compost and humic acid. Int. J. Plant Soil Sci. 8(4), 1-8. https://doi.org/10.9734/IJPSS/2015/19242 (2015).

29. Rashid, I., Murtaza, G., Zahir, Z. A. \& Farooq, G. Effect of humic and fulvic acid transformation on cadmium availability to wheat cultivars in sewage sludge amended soil to wheat cultivars in sewage sludge amended soil. Environ. Sci. Pollut. Res. 25(16), 16071-16079. https://doi.org/10.1007/s11356-018-1821-9 (2018).

30. Conte, P., Agretto, A., Spaccini, R. \& Piccolo, A. Soil remediation: Humic acids as natural surfactants in the washings of highly contaminated soils. Environ. Pollut. 135, 515-522 (2005).

31. Tan, K. H. Humic Matter in Soil and the Environment: Principles and Controversies 2nd edn. (CRC Press, 2014).

32. Shams, M., Yildirim, E., Arslan, E. \& Agar, G. Salinity induced alteration in DNA methylation pattern, enzyme activity, nutrient uptake and $\mathrm{H} 2 \mathrm{O} 2$ content in pepper (Capsicum annuum L.) cultivars. Acta Physiol. Plant. 42, 59. https://doi.org/10.1007/s11738020-03053-9 (2020).

33. Shao, H. B., Chu, L. Y., Jaleel, C. A. \& Zhao, C. X. Water-deficit stress-induced anatomical changes in higher plants. C. R. Biol. 331, 215-225. https://doi.org/10.1016/j.crvi.2008.01.002 (2008).

34. Tang, W. W. et al. Impact of humic/fulvic acid on the removal of heavy metals from aqueous solutions using nanomaterials: A review. Sci. Total Environ. https://doi.org/10.1016/j.scitotenv.2013.09.044 (2014).

35. Janos, P., Vavrova, J., Herzogova, L. \& Pilarova, V. Effects of inorganic and organic amendments on the mobility (leachability) of heavy metals in contaminated soil: a sequential extraction study. Geoderma 159(3-4), 335-341. https://doi.org/10.1016/j.geode rma.2010.08.009 (2010).

36. Topcuoglu, B. The influence of humic acids on the metal bioavailability and phytoextraction efficiency in long-term sludge applied soil. Conference on International Research on Food Security, Natural Resource Management and Rural Development. Tropentag, Gottingen, Germany, September 19-21 (2012).

37. Park, S., Kim, K., Kang, D., Yoon, H. \& Sung, K. Effects of humic acid on heavy metal uptake by herbaceous plants in soils simultaneously contaminated by petroleum hydrocarbons. Environ. Earth. Sci. 68(8), 2375-2384. https://doi.org/10.1007/s12665-0121920-8 (2012)

38. Kalis, E. J., Temminghoff, E. J. \& Weng, L. Effects of humic acid and competing cations on metal uptake by Lolium perenne. Environ. Toxicol. Chem. 25(3), 702-711. https://doi.org/10.1897/04-576R.1 (2006).

39. Borůvka, L. \& Drábek, O. Heavy metal distribution between fractions of humic substances in heavily polluted soils. Plant Soil Environ. 50(8), 339-345 (2004).

40. Rong, Q. et al. Humic acid reduces the available cadmium, copper, lead, and zinc in soil and their uptake by tobacco. Appl. Sci. 10, 1077. https://doi.org/10.3390/app10031077 (2020).

41. Jiang, Y., Yuan, J., Lu, Z., Wang, A. \& Chen, H. The effect of humic acid on species of Cu, Cd, Pb, $\mathrm{Zn}$ in sewage farm. J. Northwest Normal Univ. 41(6), 42-46 (2005).

42. Wang, Z., Cao, M., Cai, W. \& Zeng, H. The effect of humic acid and fulvic acid on adsorption-desorption behavior of copper and zinc in the yellow soil. AIP Conf. Proc. 1820, 040027. https://doi.org/10.1063/1.4977299 (2017).

43. Wu, S., Li, R., Peng, S., Liu, Q. \& Zhu, X. Effect of humic acid on transformation of soil heavy metals. IOP Conf. Series 207, 012089. https://doi.org/10.1088/1757-899X/207/1/012089 (2017).

\section{Acknowledgements}

We are very grateful to The Atatürk University, Scientific Research Projects Foundation (Project Number FBA2019-7219) and Humintech $\mathrm{GmbH}$ for generous support.

\section{Author contributions}

Conceptualization, E.Y., G.A. and M.E.; methodology, Z.A., M.E, A.D., M.T.; validation, R.K., A.D. and G.A.; investigation, E.Y.; resources, G.A, A.D and R.K.; data curation, S.A.; writing-original draft preparation, S.A.; writing-review and editing, R.K., M.T.; visualization, M.E.; supervision, M.T.; project administration, Z.A.,E.Y.; funding acquisition, E.Y., R.K, G.A, A.D and M.E All authors have read and agreed to the published version of the manuscript.

\section{Competing interests}

The authors declare no competing interests.

\section{Additional information}

Correspondence and requests for materials should be addressed to E.Y. or M.T.

Reprints and permissions information is available at www.nature.com/reprints.

Publisher's note Springer Nature remains neutral with regard to jurisdictional claims in published maps and institutional affiliations.

(c) (i) Open Access This article is licensed under a Creative Commons Attribution 4.0 International License, which permits use, sharing, adaptation, distribution and reproduction in any medium or format, as long as you give appropriate credit to the original author(s) and the source, provide a link to the Creative Commons licence, and indicate if changes were made. The images or other third party material in this article are included in the article's Creative Commons licence, unless indicated otherwise in a credit line to the material. If material is not included in the article's Creative Commons licence and your intended use is not permitted by statutory regulation or exceeds the permitted use, you will need to obtain permission directly from the copyright holder. To view a copy of this licence, visit http://creativecommons.org/licenses/by/4.0/.

(c) The Author(s) 2021, corrected publication 2021 